\title{
Effects of predictability on the welfare of captive animals
}

\author{
Lois Bassett, Hannah M. Buchanan-Smith* \\ Scottish Primate Research Group, Department of Psychology, University of Stirling, Stirling, \\ FK9 4LA, Scotland, United Kingdom
}

Available online 30 June 2006

\begin{abstract}
Variations in the predictability of a stressor have pronounced effects on the behavioural and physiological effects of stress in rats. It is reasonable to expect that variations in the predictability of husbandry routines thought to be aversive to animals might have similar effects on stress indices. Similarly, variations in the predictability of positive events, of which feeding is an obvious example, may affect welfare. This review examines the behavioural and physiological effects of the predictability of aversive and appetitive stimuli, and the application of experimental findings to animal husbandry in practice. It is argued here that two distinct but overlapping types of predictability exist. 'Temporal' predictability describes whether an event occurs at fixed or variable intervals, whereas 'signalled' predictability relates to the reliability of a signal preceding the event. This review examines the effects of each of these types of predictability in relation to positively and negatively perceived events, and examines the link between predictability and control. Recommendations are made for relatively simple and inexpensive modifications to husbandry routines that may be easy to incorporate into the schedules of busy staff yet could have a profound impact on the welfare of animals in their care.
\end{abstract}

(C) 2006 Elsevier B.V. All rights reserved.

Keywords: Animal welfare; Control; Husbandry routines; Signalled predictability; Temporal predictability

\section{Introduction}

The predictability of an event is known to affect an animal's response to it (Weinberg and Levine, 1980). Studies investigating the effects of predictability of stimuli on animal behaviour

\footnotetext{
This paper is part of the special issue entitled "Conservation, Enrichment and Animal Behaviour", Guest Edited by Dr. Ronald R. Swaisgood.

* Corresponding author. Tel.: +44 1786 467674; fax: +44 1786467641.

E-mail address: h.m.buchanan-smith@stir.ac.uk (H.M. Buchanan-Smith).
} 
and welfare have tended to manipulate predictability in one of two ways. The most obvious method involves manipulating the temporal characteristics of the stimulus presentation, delivering it to the animal on either a fixed-time or variable-time schedule. For example, a positive stimulus, such as food, or an aversive stimulus, such as electric shock, might be delivered to animals at random times, which are irregular and therefore unpredictable. Alternatively, the stimulus could be delivered at fixed times, which are regular and therefore predictable. The second method involves preceding the stimulus with a signal. A regular signal preceding the stimulus by the same time interval will render it predictable, irrespective of whether it occurs on a fixed or variable time schedule. Variations in the predictability of the stimulus may be achieved by manipulating the reliability of the signal preceding it. Thus, a stimulus occurring after $50 \%$ of signals will be less predictable than one occurring after $100 \%$ of signals.

No studies have thus far specifically discriminated between these two methods of varying the predictability of a stimulus. However, it is proposed here that the methods relate to two different types of predictability, referred to hereafter as 'temporal' and 'signalled' predictability. This review therefore discusses studies in the light of these different types of predictability.

The motivational consequences of predictability, however, are thought to be closely related to those of control. An event is deemed controllable if there is a difference in the likelihood of it occurring depending on an animal's behaviour (Overmier et al., 1980; Sambrook and BuchananSmith, 1997). Some researchers (e.g. Mineka and Hendersen, 1985) suggest that the relationship between these two factors is so profound that a full understanding of one cannot be achieved without the other. For this reason, this review will also discuss the effects of control, as well as the relationships, and potential confound, between these two variables.

\section{Measurement of welfare}

Welfare is notoriously difficult to measure (e.g. Mason and Mendl, 1993). An animal's welfare may lie on a scale from bad to good, and a combination of behavioural, physiological and biochemical measures will produce a more complete picture of an animal's welfare. There are three measures that the studies examining the effects of predictability of aversive stimuli use. First, there are those related to hypothalamic-pituitary-adrenal (HPA) activation, and measured by levels of corticosteroids. However, as described below, short-term (acute) increases in corticosteroids may be adaptive so should not always be viewed negatively. However, if levels are raised chronically, this is of concern as it impacts on normal functioning. Second, there are those relating to the physical health of the animals, such as gastric ulceration and anorexia. Gastric ulceration is less controversial as a negative welfare indicator; it suggests chronic stress. Anorexia is a well-accepted symptom of chronic intractable pain and stress, resulting in weight loss or reduced weight gain (Harris et al., 2002). Third, studies have used preference testsgiving the animals choice between, for example, predictable and unpredictable electric shock. These tests provide a clear indication of the animals' preference, but they are not without their critics (Arthur, 1986, and see Fraser and Matthews, 1997 for a review).

Whilst some studies on predictability of positive stimuli have also used preference tests, the majority have used behaviour patterns to determine welfare. Stereotypic behaviour (repetitive, unvarying and apparently functionless behaviour patterns) is often used an indicator of poor welfare (Mason and Latham, 2004). However, as they, and Mason et al. (2007) warn, there is not a one-to-one relationship, and stereotypies should never be used as the sole indicator of poor welfare (or a reduction as the sole indicator of improved welfare). Increases in abnormal behaviours (often species-specific, but such as eye-poking in macaques), self-directed behaviours 
(such as scratching), coprophagy, or agonistic behaviour (especially if injurious) can also be viewed negatively as they may be indicative of tension and frustration (Arnone and Dantzer, 1980; Castles and Whiten, 1998; Diezinger and Anderson, 1986; Maestripieri et al., 1992; de Monte et al., 1992). Increases in inactivity are also often viewed negatively but again caution must be exercised and this should only be interpreted as such in combination with other measures.

Whilst there is a broad range of behavioural and physiological measures of poor welfare described above, there are substantially fewer behaviours, and no physiological measures, that are used as indicators of good welfare in studies described in this review. The behaviours that are used to indicate improved welfare are exploratory behaviour, and an increased range of behaviours (Young, 2003), together with a reduction of behaviours indicative of poor welfare. It is therefore noted at the outset of this review that this discrepancy between measures of welfare assessment, combined with the differences in the form of the experiments concerning predictability of positive and negative stimuli, makes direct comparison between the effects of predictability on positive and negative events difficult.

\section{The behavioural and physiological welfare effects and adaptive significance of responses to predictability}

Captive animals are invariably held in an environment that is smaller and less complex than their natural habitat (Chamove and Anderson, 1989; Buchanan-Smith, 1997). Reduced environmental complexity is generally associated with an increase in predictability, as these two factors are inversely related. Many animals have evolved endogenous clocks enabling them to predict and exploit temporal regularities of environmental food availability (Mistlberger, 1994; Roberts, 1998). However, deviations from a regular and predictable schedule of food availability can and do occur. Environmental unpredictability has been described as

'a single, often acute, event such as an attack by a predator or the occurrence of a snowstorm that, for hours to days, disrupts 'normal' ongoing activities by temporally diminishing food resources and by increasing energetic demands' (Reneerkens et al., 2002, p. 81).

Studies have shown that unpredictable environmental conditions result in elevated concentrations of corticosteroids in a range of vertebrates, including mammals, birds, reptiles, amphibians and teleost fish (review by Wingfield and Ramenofsky, 1999). The long-term activation of these hormones is unlikely to be adaptive in a free-living individual, as it may result in delay of puberty, suppression of growth, metabolic exhaustion from breakdown in skeletal muscle, increased susceptibility to disease and neuron death in the hippocampus (Wingfield and Ramenofsky, 1999). However, the effects of the short-term activation of corticosteroids may be adaptive as they trigger physiological and behavioural responses to overcome the impact of the stressor, such as suppression of reproductive and territorial behaviour, as well as facilitation of foraging and exploratory behaviour (Wingfield and Ramenofsky, 1999). For example, elevations of corticosterone are associated with increased exploration in rats and white-crowned sparrows (Breuner, 1998; Sandi et al., 1996). Spatial memory is also enhanced in rats and mountain chickadees when corticosterone levels rise (Luine et al., 1996; Saldanha et al., 2000).

Temporally unpredictable feeding results in elevated levels of corticosterone in captive red knots, a shorebird, possibly due to a 'perception of uncertainty' (Reneerkens et al., 2002, p. 86). It has been proposed that this increase in corticosteroids may promote both exploratory behaviour and enhanced memory performance, which are necessary in order to find food when supplies are 
unpredictably variable. These costly processes are therefore tailored to the needs of the individual (Reneerkens et al., 2002). The responses may only be adaptive when the animal has a degree of control over his/her environment and, for example, is able to increase the amount of exploration in response to an unpredictable food supply. In a barren cage in the captive situation, however, increased exploration may be impossible. The inability to respond appropriately to stimuli with such adaptive behaviours may mean that motivation to perform these behaviours may not be reduced, resulting in welfare problems (Hughes and Duncan, 1988).

Although animals have evolved to cope with environments of great temporal and physical complexity, until recently a widely held view was that predictable captive environments were preferable because they offered security and consequently reduced stress (Shepherdson, 1989). It is probable that a human preference for routine is partly responsible for this, along with several scientific studies concerning the effects of predictability of aversive stimuli. However, there may be positive aspects of predictability which are due to control, which has been shown to be a factor capable of reducing the physiological response of animals to stressors (Weinberg and Levine, 1980).

Given that the nature (i.e. positive, neutral or negative) of the stimuli in question, and the context in which they are offered, will affect the consequences of predictability or unpredictability (van Rooijen, 1991), the review discusses positive events and negative (aversive) events separately. The discussion of positive events relate primarily to those that are appetitive (i.e. feeding) as few data are available for other positive events. The aversive events literature is almost exclusively restricted to experimental studies on the administration of electric shocks in rats. In addition the separation of positive and negative events is warranted as the methodologies used, and the measurement variables are so different that direct comparison is difficult.

\section{Experimental studies on the effects of predictability of aversive events}

A number of behavioural studies have used electric shocks as aversive stimuli, and rendered the signalled predictability of these events high (by preceding their presentation by a conditioned stimulus acting as a signal, e.g. a tone or light) or low (no signal). Rats, fish and birds offered a choice between predictable and unpredictable electric shock will generally choose shock with high signalled predictability (reviewed by Badia et al., 1979), whether or not they are able to escape it. Predictable shock has also been found to be less behaviourally disruptive than unpredictable shock (Davis and Levine, 1982; Seligman and Meyer, 1970). These results have been taken to suggest that an element of predictability makes shock less aversive. This effect is so pronounced that rats chose signalled rather than unsignalled shock even when the predictable shock was four to nine times longer and two to three times stronger than that in the unpredictable condition (Badia et al., 1973).

One study (Badia et al., 1975) looked at both temporal and signalled predictability of shock. It was found that temporally predictable shock was chosen by rats over temporally unpredictable shock when both conditions were unsignalled. However, when a signal was introduced into the temporally unpredictable condition, this preference was reversed (Badia et al., 1975). This suggests that signalled predictability may be more important, or at least more perceptible, than temporal predictability.

Studies relating predictability to physiological measures of stress, however, are less consistent in their conclusions. Unsignalled and temporally unpredictable aversive stimuli have been shown to be associated with physiological stress responses such as gastric ulcers, weight loss and increased plasma corticosterone concentrations (Gliner, 1972; Weiss, 1972; Seligman and 
Meyer, 1970). In contrast to these findings, there is also a large body of experimental evidence to suggest that signalled or temporally predictable shock is more aversive for animals, according to similar physiological stress indices (reviewed by Weinberg and Levine, 1980). Hennessy et al. (1977) altered the temporal predictability of shock by manipulating the regularity of the intershock interval. Highly temporally predictable shock, where the temporal regularity of the schedule could be used to predict the next shock presentation, was associated with a high level of HPA activation. Highly temporally unpredictable shock, where the animals had no cues to enable them to predict the next shock presentation, resulted in a similarly high level of activation of plasma corticoids. Moderately temporally unpredictable shocks, delivered on a schedule with moderate variability in the inter-shock interval, however, resulted in significantly lower HPA activation. Further, one of the few studies on predictability of aversive events that is not on rats, and did not use shock, found that infant squirrel monkeys showed greater physiological and behavioural stress responses on maternal separation of predictable duration than on that of unpredictable duration (Jordan et al., 1984).

Various explanations have been suggested for these observed effects, and the discrepancies between findings relating predictability to stress. These have focused on the following parameters.

\subsection{Study length}

Abbott et al. (1984) examined the literature on physiological responses to predictable versus unpredictable shock in an attempt to explain the conflicting results of behavioural and physiological studies on the effects of predictability. The majority of these studies had used the presence or absence of a signal to render shock predictable or unpredictable. Abbott et al. (1984) found that the length of the experiment varied considerably between studies. They concluded that unpredictable shock is more stressful than predictable shock in short-term studies, but less stressful in long-term studies. The researchers accounted for this with the following explanation. In short-term studies, unpredictable shock leads to high arousal and possible physiological exhaustion, whereas 'safe periods' associated with predictable shock provide relief from stress, making this condition less stressful overall. However, in long-term studies, lack of adaptation in the predictable shock condition may eventually cause exhaustion, which would not occur in animals adapted to the threat of unpredictable shock.

Arthur (1986) refutes the conclusions of Abbott et al. (1984), claiming that their classification of studies into short- and long-term is unsatisfactory. He claims that predictable shock is more stressful in either short- or long-term studies, and that conflicting results in previous studies are due to confounding variables. If this is true, physiological evidence indicating that predictable shock is more aversive than unpredictable shock appears to contradict the behavioural evidence, which shows that animals choose predictable over unpredictable shock, given a choice (reviewed by Badia et al., 1979). These behavioural studies with rats have been taken to mean that predictable shock is less aversive than unpredictable shock.

\subsection{Measure of stress used, and the assumption that different physiological and behavioural indices of stress are directly comparable}

Different measures are used to indicate physiological stress in the various studies, and these different physiological measures may not be directly comparable. For example, it is thought that gastric ulceration may give a different impression of stress severity as compared to a more acute indicator such as HPA activation (Weinberg and Levine, 1980). The discrepancy between 
behavioural preference for predictable over unpredictable shock (reviewed by Badia et al., 1979) and the physiological stress response which does not wholly support this conclusion suggest that behavioural and physiological measures of stress are not analogous.

\subsection{Preference tests may not necessarily be good indicators of stress}

Miller et al. (1983) trained water-deprived rats to press a 'high-aversiveness' or 'lowaversiveness' lever in response to a fixed-electrode variable-intensity tail shock, in order to obtain water. This enabled the experimenters to gauge how intense the rats perceived the shocks to be. They showed that although rats prefer signalled predictable rather than unpredictable shock, they actually perceive the former as more intense. This finding implies that preference does not necessarily indicate the stressfulness of the various conditions. Arthur (1986) claims that it is nonsensical to conclude that predictable shock is less stressful when there is evidence (Miller et al., 1983) that rats experience predictable shock as more intense. It appears, therefore, that predictable shock may be more aversive (in terms of physiological stress responses) than unpredictable shock under certain experimental conditions. Pitman et al. (1995) showed that greater predictability of shock was associated with higher plasma corticosterone and norepinephrine levels, taken to be indicative of chronic stress, supporting the conclusions of Miller et al. (1983). Pitman et al. (1995) believe that signals reliably predicting shocks cause sensitisation of central neural control of adrenocortical activity, whereas unpredictable shocks cause habituation of the central nervous system to occur. The mechanisms behind these processes are, however, unclear.

\subsection{Control}

Control has been defined, in relation to studies using aversive stimuli, as

'the ability to make active responses during an aversive stimulus' (Weinberg and Levine, 1980, p. 45).

Active responses may allow the animal to escape or avoid the stressor, which may have the effect of reducing stress responses. However, active responses may only allow the animal to move from one stimulus condition to another, rather than to escape from the stressor altogether. Even in this situation, an element of control appears to reduce the physiological stress response to aversive stimuli such as shock (Weinberg and Levine, 1980).

Study animals in many of the experiments conducted to investigate the effects of predictability also had a degree of control, although control was not mentioned as an experimental parameter (Weinberg and Levine, 1980). Many of the effects attributed to predictability in these studies may therefore be due to this potential confound. The 'preparatory response' hypothesis has been proposed to explain the apparent behavioural preference of animals for predictable over unpredictable shock (Perkins, 1955, 1968; Lockard, 1963). The hypothesis suggests that signals preceding events allow animals to prepare for these events, which may reduce the aversiveness of a negative stimulation, or, conversely, increase the positive nature of appetitive events (Badia et al., 1979). Preparation is thought to take place as a conditioned response, the biological function of which

'is to enable the animal to optimize interaction with the forthcoming biologically important event’ (Hollis, 1982, p. 3). 
For example, animals being given shocks may be able to change their posture in order to minimise these shocks.

In such experiments, what is taken to be a preference for predictable signalled shock may in fact be a preference for shock preceded by signals which enables such preparatory postural responding (Weinberg and Levine, 1980). This potential confounding factor has however been controlled for in many studies, for example in the work of Weiss (1972), that used a tail electrode to deliver shock with varying signalled predictability to rats. This was designed specifically to prevent the problem of unequal shock, as postural movements by the animals would not displace the electrode and so reduce the intensity of shock received. Weiss (1972) found that rats exposed to unpredictable tail shocks developed more gastric ulcers than those exposed to predictable shocks, suggesting that unpredictable shocks were more stressful even when preparatory postural changes were impossible. Physiological effects of control, and the predictability-control confound, will be further discussed later in this review.

\section{Feedback}

An element of relevant feedback is involved in most of the experiments on predictability (Weinberg and Levine, 1980). Relevant feedback has been defined as

'stimuli that are not associated with the stressor and that follow a response' (Weiss, 1971a, p. 10).

Thus feedback differs from signalled predictability which involves stimuli associated with the stressor but are not related to the response. Feedback provides the animal with information as to whether its response was successful in reducing or eliminating the stressor, and/or that the stressor has ceased. Animals provided with feedback, in the form of a series of tones following shock offset leading up to a beeping tone warning of shock, were shown to develop fewer gastric ulcers than those without this information (Weiss, 1971a) if the subject could avoid or escape the shock. Feedback is thought to be extremely important in determining animals' responses in aversive situations. Lack of feedback has been shown to increase physiological stress responses including gastric ulceration and HPA activity, while increased feedback may reduce these responses (Weinberg and Levine, 1980).

It has been suggested that predictability of aversive stimuli reduces stress in animals because it provides feedback about safe periods, when aversive stimuli are not likely to occur (Lockard, 1963; Seligman, 1968). This 'safety signal hypothesis' states that if an aversive stimulus, such as shock, is predicted by a signal, the absence of that signal indicates that the situation is safe, and no shock will occur. When shock is predictable, animals will be in a state of fear only when the signal is present, and not in its absence. However, when the shock is unpredictable, there is no such safety period signalled. The animal will constantly anticipate shock, and be in a chronic state of fear. Seligman and Meyer (1970) claim that this hypothesis explains the negative physiological and behavioural effects of unpredictability.

Badia et al. (1976) tested whether stress was diminished only when a shock-free period was identifiable, or whether a warning signal predicting shock had the same effect. They found that animals showed no preference for reliable over unreliable signals predicting shock. However, animals strongly preferred reliable over unreliable 'safety' signals, which identified shock-free periods. The researchers concluded that this study strongly supported the safety-signal hypothesis, in that it showed that a reliable indicator of shock-free periods was more important to the animal than a similar indicator of shocks. Several other studies have produced results 
supporting the safety-signal hypothesis (e.g. Badia et al., 1971; Badia and Culbertson, 1972; Arabian and Desiderato, 1975; Hennessy et al., 1977 but see Badia et al., 1976, 1979).

As is seen with control, feedback is a factor in many experimental studies on the effects of predictability of aversive events. Negative aspects of unpredictability may be due to lack of information about safety, which leaves the animals in a chronic state of fear, or anticipation, as they are unable to relax preparatory responses.

\section{Conclusions on aversive studies and applications for animal management and husbandry}

Studies investigating the effects of predictability of aversive events on behavioural and physiological responses are complex, confusing and often questionable in terms of experimental validity and in their generalisation to common practice. However, they consistently show that animals actively choose (both signalled and temporally) predictable over unpredictable aversive events (but see Arthur, 1986). Whilst the physiological stress response data are less consistent, and hence further research is required especially with common-place negative events, they also suggest that aversive events should be made predictable (but see Arthur, 1986; Weinberg and Levine, 1980), at least if the events are to be present over a short duration only (but see Abbott et al., 1984). Further, the evidence suggests that signalled predictability is more critical than temporal predictability.

There are a number of aversive events that occur in the life of captive animals. In laboratories, common aversive stimuli might include cage cleaning, or restraint for injection or blood draws (Line et al., 1991; Reinhardt, 2003). In zoos, animals are regularly faced with high visitor densities and noise which are known to impact adversely on the behaviour of many animals (reviewed in Hosey, 2000). In laboratories, it may be both beneficial and practical to give a reliable signal to indicate the onset of an aversive event (and thus the absence of such a signal indicates that the situation is safe). Given that it is often the same staff that will perform neutral and positive events (such as animal checking, feeding), the staff may become an unreliable signal to any such events. The use of a unique signal to indicate an aversive event may therefore be beneficial in laboratories carrying out regular aversive procedures, although to our knowledge no studies have specifically examined how this impacts on welfare. To determine whether the findings from experimental studies with electric shocks in rats can be applied to more typical events in captive situations, it is recommended that one aversive event (perceived to be the most aversive) is chosen and that the behaviour both before the event and following the event is compared between baseline (unsignalled) and when a signal has been introduced. Physiological responses to the unsignalled and signalled event should also be compared. However, given that animals readily associate signals (e.g. noise and visual stimuli such as specific people, clothes, a trolley, number of technicians, etc.) with events, it may be that they are already receiving unintentional signals of the imminent event, and the nature of it. If these signals are unreliable, attempts should be made to remove them.

There are also a number of less frequent aversive events that captive animals experience. For example, farm animals, such as sheep are caught annually for shearing, or dipping, and captive animals (including pets) occasionally undergo capture and restraint for veterinary procedures. One negative association is often sufficient to induce fear and animals have long memories, especially for those associated with negative experiences. Thus a clear signal may also be appropriate in such circumstances. However, caregivers should be cautious that the signal only refers to the individual for whom the event will take place (i.e. do not alert a whole group to an 
aversive event that will only take place for one individual). This may be achieved by the use of a specific signal for each animal (i.e. use his/her name, have eye contact with only the animal who will experience the event, etc.).

\section{Negative and positive and reinforcement training}

One way that control and predictability can be increased for aversive events is through positive reinforcement training (PRT), and both PRT and negative reinforcement training (NRT) are widely used in husbandry and management, sometimes unintentionally. Both positive reinforcement training (PRT) and negative reinforcement training (NRT) rely on the principles of operant conditioning in which animals learn associations between their own behaviour and the consequences of performing that behaviour. The ability of the animal to learn about the consequences of their actions and therefore to have control over his/her environment has the potential to greatly improve welfare (see below).

Negative reinforcement training (NRT) is commonly used to induce co-operation (Laule, 1999), for example for venipuncture in primates housed in laboratories or for shifting location. During NRT, the animal learns to perform a behaviour in order to avoid an aversive stimulus. The negative reinforcer may be anything from electric shock, as was used in early studies of operant learning (e.g. Garcia and Koelling, 1966), to the use of a squeeze back (Reinhardt, 2003), to the threat of capture with a net or loud noises (Phillippi-Falkenstein and Clarke, 1992). Negative reinforcement increases the performance of a desired behaviour and should not be confused with punishment, where the aversive stimulus is applied after the performance of a behaviour in order to reduce the likelihood of its reoccurrence. The scope of NRT to improve welfare is limited as the animal learns to co-operate in order to avoid an even more aversive stimulus. Thus, the choice is forced, real control is limited and the animal is subject to stress from both the procedure and the threat of the aversive stimulus used to enforce co-operation.

In contrast, with PRT rewards are used to increase the performance of a preceding behaviour. Thus, by association, the animal learns to perform a certain behaviour or series of behaviours, in response to a cue from the trainer, in order to receive something desirable, for example a preferred item of food, verbal praise, a preferred toy or social access (Laule, 1999). Unlike NRT, PRT relies on the voluntary co-operation of the subject in the procedure and the animal is therefore provided with far greater control (and hence predictability) over the event than those trained using NRT. For this reason it has been proposed that the process of training itself can be rewarding, as animals voluntarily take part and must work in order to obtain rewards and develop cognitive skills (Scott, 1990; Laule and Desmond, 1998).

For aversive events, it may not be practical to train solely using PRT. In these cases, if NRT is used it should be in combination with PRT, which allows some degree of predictability and is preferable to alternatives that often use force or restraint (e.g. Reinhardt, 2003). The integration of PRT programmes into animal management regimes has not yet been fully exploited, yet it has considerable potential to improve the care and well-being of animals in captivity (e.g. see Prescott and Buchanan-Smith, 2003).

\section{Experimental studies on the effects of predictability of appetitive events}

Many events in the life of captive animals are likely to be positive, such as feeding, provision of enrichment, access to conspecifics or other parts of the enclosure. Feeding is an event that is likely to be of great positive significance in the routine of a captive animal, and nearly all the 
studies on predictability have been carried out to investigate the effects of manipulating the predictability of food provisioning. An additional element of predictability must be introduced here, that of spatial unpredictability where food is delivered in different locations. Varying spatial predictability is analogous to feeding in the wild, although the most appropriate degree of predictability will depend upon the species' feeding ecology. Many environmental enrichment techniques use the fact that food is naturally reinforcing (extrinsic reinforcement, see Tarou and Bashaw, 2007) and interesting to animals to stimulate a range of manipulative and exploratory acts (Lindburg, 1998). In these cases, feeding is contingent up on the animal's own behaviour rather than that of the caregivers. An exception is the case of PRT (where rewards are contingent upon both the animal's behaviour and that of the caregiver/trainer), thus increasing the animal's control, and predictability over the rewards.

Early studies showed that pigeons (Wykoff, 1952) and rats (Prokasky, 1956) showed a consistent preference for conditions in which they were able to use signals to predict the presence or absence of food, compared with conditions where food delivery could not be anticipated. This was despite the fact that the average amount of food delivered in each condition was the same. Prokasky (1956) suggested that the preference for predictable over unpredictable food delivery might be due to the enabling of preparatory responses, such as salivation, to occur when food delivery could be anticipated. When electric brain stimulation was used as a reinforcer, it was also found that rats preferred signalled over unsignalled reinforcement (Cantor and LoLordo, 1970). The 'preparatory response' hypothesis, already mentioned in relation to animals' apparent preference for signalled over unsignalled shock (Perkins, 1955, 1968; Lockard, 1963) is also applicable to appetitive stimuli. It is thought that preparation for positive events may increase the reinforcing nature of such events (Badia et al., 1979). For example, when applied to feeding, a signal allowing the animal to predict food delivery might allow it to salivate. Food plus salivation is thought to be more reinforcing than food in the absence of salivation. Similarly, when food is not delivered, no anticipatory salivation plus no food is more reinforcing than salivation plus no food (Perkins, 1955, 1968; Badia et al., 1979). Thus, the weight of evidence suggests that as with aversive events, animals also prefer signalled predictability to unsignalled predictability for appetitive events.

By contrast, evidence suggests that temporal unpredictability may enhance welfare. Shepherdson et al. (1993) changed the feeding routine of three leopard cats to an unpredictable temporal schedule, and also made the food spatially unpredictable by hiding it in various places around the enclosure. Stereotypic behaviour was consequently reduced, exploratory behaviour increased and a greater range of behaviour was seen. All these changes were interpreted as being beneficial for welfare. A similar reduction in stereotyped behaviour was reported by Jenny and Schmid (2002) who introduced a less predictable feeding regime (both temporally and in location) to Amur tigers. The consequences of temporal and spatial predictability are confounded in these studies.

Other studies have addressed the question of whether feeding on an unpredictable time schedule may improve welfare. Studies have shown a variety of species to possess the ability to estimate time intervals (Richelle and Lejeune, 1980). The capability to detect, learn and use temporal information about events, stimuli, responses and rewards is thought to be a basic and adaptive aspect of animal behaviour (Higa and Staddon, 1997). This kind of information may play an important role in foraging strategies, allowing animals to estimate intervals between food availability and acquisition in a particular patch. This would enable cost-benefit judgements to be made regarding moving to different areas that might yield more abundant food supplies (Taylor et al., 2002). 
Mason (1993) argues that locomotor stereotypies may develop from appetitive foraging behaviour. Temporally predictable feeding schedules have been linked to stereotypies in some carnivores (Carlstead, 1998) and to vocalization and stereotypy in François langurs (Krishnamurthy, 1994). Indeed, predictable feeding routines have been linked to 'foodanticipatory activity' (FAA), characterised by increased arousal and activity, and documented in rodents, bees, fish, birds, rabbits, mammalian carnivores and some primate species including squirrel monkeys (reviewed by Mistlberger, 1994).

Frequently observed anticipatory activities (i.e. adjunctive behaviours (schedule-induced behaviours) described by Falk $(1971,1977)$ ) include wheel running in rodents, unreinforced lever pressing, activity directed at the empty feeding trough, general cage activity and drinking (Mistlberger, 1994). These behaviours are thought to be classically conditioned through repeated pairings of the circadian phase with food presentation (Armstrong, 1980). FAA is generally, but not exclusively, seen in animals fed on a regular daily schedule, where food availability is restricted. The FAA seen in food restricted animals therefore constitutes an additional welfare concern to this practice.

Interestingly, when previously food-restricted animals are fed ad libitum, FAA dissipates and is generally absent after 3-4 days (Mistlberger, 1994). However, to avoid excessive weight gain, ad libitum feeding will require that animals are fed larger quantities of lower calorie (but still appetitively rewarding) food or that consumption be slowed by requiring animals to spend time processing or otherwise "working" for food. The FAA phenomenon has also been seen in rats fed standard lab chow ad libitum, but given a supplementary food of a chocolate mash that was presumably highly palatable (Mistlberger and Rusak, 1987). Therefore, FAA is also relevant to other species in response to prized food items even when standard food is constantly available. As Falk (1977) points out, the expression of adjunctive behaviour across species is likely to be related to their feeding ecology.

Anticipatory behaviour has been reported in a number of primates. Increased agonistic behaviour has been observed in chimpanzees (de Waal and Hoekstra, 1980; Reynolds and Luscombe, 1969; Wilson and Wilson, 1968) and hamadryas baboons (Wasserman and Cruikshank, 1983) during pre-feeding periods when animals were fed on a predictable temporal schedule. Stump-tailed macaques showed significant increases in rates of self-directed behaviour, inactivity, vocalisation and abnormal behaviours prior to feeding (Waitt and Buchanan-Smith, 2001). Captive chimpanzees showed increased inactivity and coprophagy prior to feeding on a predictable temporal schedule (Bloomsmith and Lambeth, 1995). Such behaviour, although differing from the arousal and activity definition of food-anticipatory activity, still indicates that the animals are in anticipation of feeding. The authors claim that

'it seemed that the subjects were "waiting" for the meal to be fed' (Bloomsmith and Lambeth, 1995, p. 71).

Johannesson and Ladewig (2000) suggest that in a very predictable environment, animals may become locked into cycles of anticipating the regularly occurring events, while individuals in less predictable environments experience higher motivation for exploration and foraging. Of course, this may only be of benefit to welfare if the environment in which the animal is housed allows for such increased exploration and foraging. Bloomsmith and Lambeth (1995) found that feeding chimpanzees on an unpredictable temporal schedule led to an increase in speciesappropriate behaviour, which they suggested was indicative of improved welfare. Bloomsmith and Lambeth (1995) wrote that their results supported Jordan et al.'s (1984) proposal that, in the absence of control, predictability may be more stressful than unpredictability. 
In the study by Bloomsmith and Lambeth (1995), although chimpanzees received food on an unpredictable temporal schedule, they did receive a certain amount of signalled information relating to food delivery. Food was delivered to the different groups of chimpanzees by keepers standing at the roof level of the enclosures, who were visible to some members of the colony. These animals tended to respond with food vocalisations, which acted as signals to the rest of the colony that food delivery was imminent. However, as the chimpanzees being fed on an unpredictable schedule were typically not fed at the same time as the other groups, these signals were unreliable. Therefore, in addition to temporal predictability being low, an unreliable signal was present, although this variable was not considered by the authors. The behaviors attributed to low temporal predictability may therefore have been affected by the feeding-related signals available to the animals.

The importance of removing unreliable signals associated with feeding, and replacing them with a unique reliable signal has been demonstrated by Bassett (2003) in a study on stump-tailed macaques housed in a laboratory. Behavioural observations were made around feeding time in the presence of naturally occurring unreliable signals, and then again when these signals had been, as far as possible, removed. An artificial reliable signal was then introduced for a further period of observations, and finally, that signal was rendered unreliable by withholding food after it. Behaviour was compared in the four conditions. Food delivery preceded by an unreliable (naturally occurring or artificial) signal was associated with high levels of self-scratching. The removal of unreliable signals, as well as the introduction of a reliable signal, resulted in decreases in self-scratching, which suggests a reduction in stress in the study animals (Castles and Whiten, 1998; Diezinger and Anderson, 1986; Maestripieri et al., 1992).

Carlstead (1986) manipulated the signalled predictability of feeding by changing the reliability of signals (in the form of a bell) announcing the arrival of food to pigs. Food was delivered, from an automated hopper, on an unpredictable temporal schedule with the bell being the only information available to the pigs concerning the onset of feeding. The animals initially received food preceded by reliable signals. However, when these signals became unreliable, low predictability was found to be associated with frustration, which led to aggression and increased competition for food.

In a second experiment, pigs consistently receiving unreliable feeding signals showed a significant increase in aggressive interactions, mainly following unexpected disturbances in the environment. The author suggests that this increase was because pigs exposed to unreliable feeding signals treated these unexpected environmental noises as potential feeding signals. The failure of these 'signals' to be followed by food led to increased frustration and aggression. This did not occur in pigs which received reliable signals, however, as there was only one unmistakable signal associated with feeding, and therefore unexpected noises were not responded to as unreliable indicators of feeding time (Carlstead, 1986). The results of this study may be viewed as in accordance with the 'safety-signal hypothesis' (Seligman, 1968; Seligman and Meyer, 1970). The unambiguous, reliable feeding signal provided information for the pigs regarding 'safe' periods when the intensely stimulating event, feeding, would not occur. Carlstead (1986) claims that feeding animals on a predictable temporal schedule will not provide enough information for them regarding 'safe' period; she states that

'the presence of an unmistakable signal is the important factor for predictability' (p. 35).

It is unclear from this study whether pigs experiencing a loss of signalled predictability experienced more frustration than those that had been exposed to unreliable feeding signals from the start of the study (Carlstead, 1986, see below). 


\section{Conclusions on appetitive studies and applications for animal management and husbandry}

Although feeding is clearly essential to the physical well-being of animals, the effects of its predictability on psychological well-being has rarely been directly addressed (but see, e.g. Bassett, 2003; Bloomsmith and Lambeth, 1995; Carlstead, 1986; Waitt and Buchanan-Smith, 2001; Waitt et al., 2001 for exceptions). The complexity of the literature leaves animal caregivers confused as to whether temporally predictable feeding schedules are good because they offer the animal security or bad because they lead to FAA. The conclusion from this review, combining theory and empirical results is that animals should be fed on unpredictable feeding schedules if possible (although note that the benefits have only been demonstrated in a limited range of species). In addition, unreliable signals relating to feeding should be eliminated, and a unique reliable signal introduced.

The rationale behind this conclusion is that in the captive situation, there will always be certain signals associated with feeding, such as the sound of food preparation, doors being unlocked or other animals being fed. These signals may not always be reliable, especially in situations where there are many animals and therefore many feeding-related signals. Unreliable signals may lead to frustration, but it is unrealistic to expect these signals to be eliminated. In these situations, it may be useful for animals to learn to associate a unique noise, such as a buzzer or bell, with feeding. This sound would only be heard prior to feeding, and may help to extinguish previously learned signal associations as described above, which may not be reliable. It may be possible, using such a method, to feed on an unpredictable temporal schedule and derive benefits such as those seen by Bloomsmith and Lambeth (1995) (i.e. increased species-appropriate behaviour), but without the negative consequences observed by Carlstead (1986) (i.e. aggression).

\section{Effects of loss of predictability on animal welfare}

This review has described studies whose body of evidence shows that (signalled and temporal) predictable aversive events (electric shock) are less deleterious than unpredictable events (but see Weinberg and Levine, 1980). However, some researchers (e.g. Mineka and Kihlstrom, 1978; Tsuda et al., 1984) have suggested that loss of predictability might produce more severe effects in animals that have had prior experience with predictable shock than in individuals that have never been exposed to predictable stimuli. In other words, loss of predictability would be more detrimental to welfare than lack of predictability (unpredictability). This hypothesis is largely due to extrapolation of findings from studies indicating that loss of control over an aversive outcome, in animals that have previously been able to control it, is more stressful than never having had control (Hanson et al., 1976; Seligman, 1975; Tsuda et al., 1983; Weiss, 1971b). However, there was no difference in the amount and severity of gastric lesions shown by rats exposed to a loss of predictability of shock, compared to those that were continuously exposed to unpredictable shock (Tsuda et al., 1984). These results suggest that loss of controllability of shock is more deleterious in terms of stress and gastric pathogenesis than is loss of predictability. In contrast to this, Waitt et al. (2001) found that delayed cleaning routines, in which a previously temporally predictable event became unpredictable, resulted in increases in agonistic and abnormal behaviours in stump-tailed macaques. Captive primates are thought not to habituate to daily cage cleaning easily, which is considered to be at least mildly stressful to them (Line et al., 1991). Aggressive and abnormal behaviours are thought to increase in situations associated with 
tension and frustration (Arnone and Dantzer, 1980; Castles and Whiten, 1998; de Monte et al., 1992). The study by Waitt et al., 2001 therefore supports the idea that loss of predictability of an aversive event, caused by delays to an otherwise temporally predictable routine, may be stressful to animals.

The effect of loss of reliable signalled predictability on behaviour was described in an early study by Shenger-Krestovnikova (described by Pavlov, 1927). A hungry dog was given food in the presence of one conditioned visual stimulus (CS) (a circle), but not in the presence of another CS (an ellipse). The circle was therefore a reliable signal that food would be delivered. The shapes were manipulated so that the ellipse became more circular, which made discrimination between the two shapes progressively more difficult. Eventually the dog was unable to predict reliably whether the CS would be followed by food, and food delivery was therefore unpredictable. Behavioural changes such as squealing, wriggling and violent barking were seen at this point in the study. Pavlov describes it as presenting

'all the symptoms of a condition of acute neurosis' (p. 291).

When the discrimination between the two shapes was made easy again, the behavioural disturbances disappeared. Mineka and Kihlstrom (1978) speculate that the important variable resulting in this behavioural disturbance was loss of predictability of the food delivery in an animal that had once possessed it. The Shenger-Krestovnikova study clearly suffers from many flaws, such as a small sample size. However, it does suggest that loss of predictability, as opposed to lack of predictability, of an appetitive stimulus may have severe consequences for animal welfare.

It has been proposed that the emotion of 'hope' is elicited by situations previously paired with pleasure (Mowrer, 1960). However, when the expected reward is not delivered, the motivation of hope is aroused but not fulfilled. This results in the aversive state of 'disappointment' (Adelman and Maatsch, 1956; Wagner, 1959). Disappointment, as a result of changes in reinforcement contingencies, results in the activation of the hypothalamic-pituitary-adrenal (HPA) system (Levine et al., 1972). Thirsty rats were trained to press a lever on a variable interval (unpredictable) or a fixed interval (predictable) schedule in order to receive a single reinforcement, consisting of water (Levine et al., 1972). The conditions were then reversed. Rats experiencing an unpredictable schedule that had previously been exposed to a predictable schedule (i.e. experiencing a loss of predictability) showed a significant elevation of plasma corticoids. In contrast, those that were changed from an unpredictable to a predictable schedule did not show increased HPA activity.

Although it is debatable whether loss of predictability of an aversive event is more stressful than lack of predictability, loss of predictability of an appetitive event may be more deleterious to welfare than constant unpredictability. This would have important implications for welfare if, for example, animals accustomed to a predictable feeding schedule experience disruptions to this routine so that feeding is delayed.

Dairy calves, fed on a temporally predictable schedule, showed various behavioural deviations when exposed to a 3-h delay to feeding (Johannesson and Ladewig, 2000). These behavioural changes were attributed by the authors to frustration when the calves' expectations were not fulfilled. Stump-tailed macaques showed increases in self-directed, agonistic and abnormal behaviours when their first meal of the day, consisting of a single piece of fruit, was delayed, representing a loss of temporal predictability (Waitt et al., 2001). This was despite the fact that there was still generally a considerable amount of food remaining scattered in the wood chip floor covering from the previous day. The same animals showed increases in rates of 
self-directed behaviours, inactivity, vocalisations and abnormal behaviours prior to receiving their main feed in the afternoon (Waitt and Buchanan-Smith, 2001). However, there was no significant difference in rates of these behaviours when feeding was on time compared with when it was delayed. These results are contradictory, as a significant increase in self-directed, agonistic and abnormal behaviours was found when the first feed of the day was delayed, yet a delay in the second feed was not associated with a similar significant increase in these behaviours. The perceived appetitive value may have been greater for the first (fruit) feed, together with the longer interval between the first feed and the previous one, compared with the shorter interval between the morning and afternoon feeds. However, even if delays do not cause an increase in stressrelated behaviours, delayed feeding may still result in reduced welfare as these behaviours were prolonged in the second study when feeding was behind schedule. Delayed feeding therefore may or may not result in higher levels of stress for captive animals, but it is likely to cause the inevitable tension associated with feeding on a predictable schedule to be extended.

Waitt and Buchanan-Smith (2001) suggested that the negative consequences of delays may not just be due to a loss of temporal predictability, but also to the loss of reliability of external signals accompanying daily husbandry routines. When delays occur, signals such as the sounds of food preparation may still be perceived yet not followed by the expected feed. In such cases there is also a loss of signalled predictability, which may result in behavioural frustration, as was found in pigs exposed to unreliable feeding signals by Carlstead (1986). However, as yet, no studies have separated the effects of signalled and temporal predictability of appetitive events in an attempt to tease apart their relative importance to animals.

\section{Effects of control on animal welfare}

Control is thought to be psychologically and physiologically important to animals (Chamove and Anderson, 1989; Hanson et al., 1976; Mineka et al., 1986; Overmier et al., 1980) but although the concept of control over positive stimuli is relatively well developed in the enrichment literature (e.g. Buchanan-Smith, 1997) its application is primarily limited to feeding (e.g. Line et al., 1990; Markowitz, 1982), music (Line et al., 1990) and joystick/video tasks (e.g. Lambeth et al., 2001). An event is deemed controllable if there is a difference in the likelihood of it occurring depending on an animal's behaviour (Overmier et al., 1980; Sambrook and BuchananSmith, 1997). Experimental evidence suggests that animals have a preference for control (Overmier et al., 1980). For example, deer mice, which may have an aversion to bright lighting, will use a lever to turn off a light when it comes on automatically at regular intervals (Kavanau, 1963). Their preference for control appears to override their aversion to bright light, as they will also turn it back on if it is automatically turned off. Kavanau concludes that the animals find it rewarding to exercise a degree of control over their environment. The degree of behavioural control that an animal has over a stressor is also thought to regulate the behavioural and physiological impact of that stressor (e.g. Anisman et al., 1981; Maier, 1984). For example, rats able to press a bar to avoid electric shock showed fewer physiological signs of stress, such as weight loss and gastric lesions than those that could not exercise control, even though the groups were yoked and received exactly the same amount of shock (Weiss, 1968). Positive behavioural and physiological changes have been found when captive primates have been given control over aspects of their environment such as food delivery (Line et al., 1991) or auditory stimuli (Hanson et al., 1976).

Control is thought to be so important to animals because it is the major adaptive aspect of their behaviour (Sambrook and Buchanan-Smith, 1997). In order to survive and reproduce effectively, an animal must exert control over what he/she eats, what eats (or does not eat) it, with whom he/ 
she mates and so on. Captive animals inevitably experience reduced control over their environment, compared with their wild counterparts (Chamove and Anderson, 1989). This reduced sense of control may be the aspect of captivity that animals find most stressful and therefore is most detrimental to their welfare (Markowitz, 1982).

\section{The relationship between predictability and control}

Many experiments investigating the effects of predictability and control have confounded controllability with predictability (Overmier et al., 1980). In these studies, events that were uncontrollable also tended to be unpredictable, and those that were controllable were also predictable. Conversely, one of the reasons for the contrasting results in studies of the effects of predictability may be that many of these studies have included various opportunities for control and feedback (Weinberg and Levine, 1980).

The traditional view of control makes the presumption that control cannot be present without predictability - an event may be predicted without being controlled, but may not be controlled without also being predicted (Nickels et al., 1992).

'Control is confounded by predictability in that having control over a stimulus also means that it is predictable' (Schulz, 1976, p. 564).

The motivational consequences of predictability and control are thought to be closely related; some researchers suggest that this relationship is so intimate that a full understanding of these effects will only be achieved by examining them both together (Mineka and Hendersen, 1985). Several theories have been proposed to explain the relationships and interactions between predictability and controllability.

\subsection{The effects of predictability and controllability are additive}

Weiss (1971a) managed to separate the two variables, and found that absence of controllability and absence of predictability both increased the incidence of gastric lesions in the rat. The effects of uncontrollability and unpredictability also appeared to be additive in terms of this physiological measure. It is however, still unclear whether these two variables result in additive behavioural effects (Overmier et al., 1980).

\subsection{Control is important to organisms because it provides predictability}

Many effects that were initially attributed to control may be due to the predictability and consequent reduction of uncertainty inherent in many of the situations used to examine the effects of control (Averill, 1973). Burger and Arkin (1980) showed that predictability in the absence of control was as effective at reducing stress as predictability and control combined. However, other studies (reviewed by Miller, 1979) indicate that in some situations, predictability without control over the onset of events does not appear to be as beneficial as predictability with control. Dess et al. (1983) showed that at least in some situations, the effects of controllability and predictability are different and separate.

\subsection{Predictability is important because it allows efficient control}

This view, the converse of that suggested by Averill (1973), argues that preference for predictability is due to its enabling the individual to exert a degree of control over the stimulus 
(Biederman and Furedy, 1970, 1973, 1976; Furedy and Biederman, 1976; Lykken and Tellegen, 1974). For example, preference for signalled shock was found to emerge when rats in a shuttlebox developed the capacity to modify the shock they received by spending time on grids of the same polarity during the delivery of the signalled shock (Furedy and Biederman, 1976).

Although the three above models describe different relationships between predictability and controllability, they all make the same predictions for the outcome of experiments designed to separate the two factors (Overmier et al., 1980). The first, additive, model would predict the greatest behavioural or physiological deficits to occur in animals exposed to a stimulus that could be neither predicted nor controlled. Intermediate levels of these deficits should be seen when the stimulus is either predictable or controllable. Animals that could predict and control the stimulus should show the least behavioural or physiological disruption.

If, as suggested by the second and third models, predictability and controllability can be reduced to a single common factor, the same predictions still hold. Animals exposed to stimuli that they can neither predict nor control will experience the lowest levels of this single underlying factor, whereas those that can predict or control stimuli will experience intermediate levels. Animals that are able to both predict and control stimuli will experience the highest levels of the common factor. The fact that the three models generate identical predictions means that it is highly unlikely that researchers will be able to find experimental evidence to differentially support any of them (Overmier et al., 1980).

\section{Animal welfare implications}

Predictability and control are inextricably linked and therefore the animal welfare implications will address the effects of both. One major difference between the environments of captive and wild animals is the reduced amount of environmental control available to them, and the increased amount of predictability (Carlstead, 1996). It has been suggested by various behavioural theorists (e.g. Archer, 1976; Inglis, 1983; Salzen, 1962) that the degree to which an animal is stimulated by an event or situation is dependent on the discrepancy between his/her expectations of stimulation and the actual stimulation he/she receives. Additionally, these theorists propose that the immediate psychological goal of behaviour is to control the level of stimulation the animal receives from his/her surroundings. Animals in nature are able to control the amount of stimulation they receive, by various behaviours such as approaching, attacking or hiding from the stimulus, until the stimulation they receive is at an acceptable level, or his/her expectations of the stimulation are met. They can control thermal stimulation by moving, for example, into the sun or away from the wind. They can also satisfy appetitive motivation by, for example, seeking food or a mate (Carlstead, 1996; Buchanan-Smith, 1997).

In their review, Weinberg and Levine (1980) conclude that giving an animal an element of control over a predictable shock situation appears to make the situation less aversive. Although many events in the lives of captive animals are likely to be positive, some events, such as cage cleaning or laboratory procedures including the giving of injections, may be perceived as being of an aversive nature. Providing animals with a degree of control over these events may reduce the stress associated with them. Training, using positive reinforcement techniques, is one such way to provide animals with a degree of control.

However, for practical reasons it is likely to be impossible to enable animals to have control over many stimuli. Although some researchers have given animals control over music (e.g. Line et al., 1990), it should be noted that only one animal is likely to have control at any one time, and 
yet the consequences of his/her actions may affect all those in the group/colony room. Further the literature on loss of control shows that it may be even more detrimental than never having control so any studies providing such opportunities should take this into account. If providing control is impractical, making these events as predictable as possible may provide a viable alternative, minimising the stress associated with a lack of control. Such predictability could provide animals with information regarding 'safety periods', when negatively perceived events would not occur, enabling them to relax rather than being in a constant state of anticipation of the events (Seligman, 1968; Seligman and Meyer, 1970). Manipulating the temporal predictability of aversive events, or introducing 'safety' signals, may be an easily implemented method of reducing their impact on welfare.

Studies conducted during the last decade, however, indicate that rather than being beneficial for welfare, highly predictable environments may in themselves be stressful. This is thought to be because they may cause animals adapted to an unpredictable natural environment to become bored (van Rooijen, 1991; Wiepkema and Koolhaas, 1993). It has been suggested that, for optimal welfare, predictability of environmental events should be of an intermediate value, although this suggestion has not been substantiated (Novak and Drewson, 1989; Wiepkema and Koolhaas, 1993). Research is necessary to investigate the effects of predictability of positive, negative and neutral events in the lives of captive animals. It is also necessary to identify which events are most important to animals in terms of variations in predictability. A major challenge for research in this area is to identify optimal levels of predictability in order to enhance the welfare of captive animals.

\section{Recommendations for animal management and husbandry}

(1) There should be a thorough evaluation of both positive and negative husbandry and management routines in terms of temporal and (often unintentional) signalled predictability, and welfare should be assessed prior to positive and negative events.

(2) If possible, negative (aversive) events should be made temporally predictable, and any unreliable (often unintentional) signals should be removed where feasible.

(3) A unique and reliable signal to indicate the onset of an aversive event should also be introduced to decrease stress, but the beneficial effects should be scientifically demonstrated before widespread practice is adopted. Care must be taken to ensure the signal only refers to the individual for whom the event will take place, and not be a (unreliable) signal for the whole group.

(4) Training animals can be a useful management tool to provide improved care of captive animals. Positive reinforcement training increases the control and predictability the animals have and should be used to provide welfare benefits.

(5) Every effort should be made to avoid delays to events occurring on a (usually) reliable temporally predictable schedule, as delays (loss of temporal predictability) have a negative impact on welfare.

(6) FAA (some of which is undesirable) is primarily seen with animals which are food restricted (and fed on a predictable schedule), constituting an additional welfare concern to this practice.

(7) If possible, temporally unpredictable feeding should be introduced (although note that the benefits have only been demonstrated in a limited range of species). Unreliable signals relating to feeding should be eliminated if possible, and a unique reliable signal introduced. 


\section{Acknowledgements}

We thank Ron Swaisgood for the invitation to contribute to this Special issue, for his enthusiasm for our manuscript, his friendly and accommodating editing style and his helpful comments on how to improve it. We also thank Jean McKinley, Anna Dudek and two anonymous reviewers for useful comments. LB was funded by the Biotechnology and Biological Sciences Research Council (BBSRC).

\section{References}

Abbott, B.B., Schoen, L.S., Badia, P., 1984. Predictable and unpredictable shock: behavioral measures of aversion and physiological measures of stress. Psychol. Bull. 96, 45-71.

Adelman, H.M., Maatsch, J.L., 1956. Learning and extinction based upon frustration, food reward, and exploratory tendency. J. Exp. Psychol. 52, 311-315.

Anisman, H., Kokkinidis, L., Sklar, L.S., 1981. Contribution of neurochemical change to stress-induced behavioral deficits. In: Cooper, S.J. (Ed.), Theory in Psychopharmacology. Academic Press, London, UK.

Arabian, J.M., Desiderato, O., 1975. Preference for signaled shock: a test of two hypotheses. Anim. Learn. Behav. 3, 191195.

Archer, J., 1976. The organization of aggression and fear in vertebrates. In: Bateson, P.P.G., Klopfer, P.H. (Eds.), Perspectives in Ethology. Plenum Press, New York, USA, pp. 231-298.

Armstrong, S., 1980. A chronometric approach to the study of feeding behaviour. Neurosci. Biobehav. R. 4, $27-53$.

Arnone, M., Dantzer, R., 1980. Does frustration induce aggression in pigs? Appl. Anim. Ethol. 6, 351-362.

Arthur, A.Z., 1986. Stress of predictable and unpredictable shock. Psychol. Bull. 100, 379-383.

Averill, J.R., 1973. Personal control over aversive stimuli and its relationship to stress. Psychol. Bull. 80, $286-303$.

Badia, P., Culbertson, S.A., 1972. The relative aversiveness of signaled vs. unsignaled escapable and inescapable shock. J. Exp. Anal. Behav. 17, 463-471.

Badia, P., Culbertson, S.A., Lewis, P., 1971. The relative aversiveness of signaled vs. unsignaled avoidance. J. Exp. Anal. Behav. 16, 113-121.

Badia, P., Harsh, J., Coker, C.C., Abbot, B., 1976. Choice and the dependability of stimuli that predict shock and safety. J. Exp. Anal. Behav. 26, 95-111.

Badia, P., Culbertson, S., Harsh, J., 1973. Choice of longer or stronger signaled shock over shorter or weaker unsignaled shock. J. Exp. Anal. Behav. 19, 25-32.

Badia, P., Harsh, J., Abbott, B., 1979. Choosing between predictable and unpredictable shock conditions: data and theory. Psychol. Bull. 86, 1107-1131.

Badia, P., Harsh, J., Coker, C.C., 1975. Subjects choose fixed time over variable time shock and vice versa: shock distribution and shock-free time as factors. Learn. Motiv. 6, 264-272.

Bassett, L., 2003. Effects of Predictability of Feeding Routines on the Behaviour and Welfare of Captive Primates. Unpublished PhD dissertation, University of Stirling, Scotland.

Biederman, G.B., Furedy, J.J., 1970. The preference-for-signalled-shock phenomenon: signalling shock is reinforcing only if shock is modifiable. Q. J. Exp. Psychol. 22, 681-685.

Biederman, G.B., Furedy, J.J., 1973. Preference-for-signalled-shock phenomenon: effects of shock modifiability and light reinforcement. J. Exp. Psychol. 100, 380-386.

Biederman, G.B., Furedy, J.J., 1976. Preference for signalled shock in rats? Instrumentation and methodological errors in the archival literature. Psychol. Rec. 26, 501-514.

Bloomsmith, M.A., Lambeth, S.P., 1995. Effects of predictable versus unpredictable feeding schedules on chimpanzee behavior. Appl. Anim. Behav. Sci. 44, 65-74.

Breuner, C.W., 1998. The avian stress response: corticosterone and behavior in a wild, seasonal vertebrate. PhD Thesis, University of Washington.

Buchanan-Smith, H.M., 1997. Environmental control; an important feature of good captive callitrichid environments. In: Pryce, C., Scott, L., Schnell, C. (Eds.), Marmosets and Tamarins in Biological and Biomedical Research. DSSD Imagery, Salisbury, UK, pp. 47-53.

Burger, J.M., Arkin, R.M., 1980. Prediction, control and learned helplessness. J. Pers. Soc. Psychol. 38, $482-491$.

Cantor, M.B., LoLordo, V.M., 1970. Rats prefer signaled reinforcing brain stimulation to unsignaled USB. J. Comp. Physiol. Psychol. 71, 183-191. 
Carlstead, K., 1986. Predictability of feeding: its effects on agonistic behaviour and growth in grower pigs. Appl. Anim. Behav. Sci. 16, 25-38.

Carlstead, K., 1996. Effects of captivity on the behavior of wild mammals. In: Kleiman, D.G., Allen, M.E., Thompson, K.V., Lumpkin, S. (Eds.), Wild Mammals in Captivity: Principles and Techniques. University of Chicago Press, Chicago, USA, pp. 317-333.

Carlstead, K., 1998. Determining the causes of stereotypic behaviors in zoo carnivores: toward appropriate enrichment strategies. In: Shepherdson, D.J., Mellen, J.D., Hutchins, M. (Eds.), Second Nature: Environmental Enrichment for Captive Animals. Smithsonian Institute Press, Washington, pp. 172-183.

Castles, D.L., Whiten, A., 1998. Post-conflict behaviour of wild olive baboons. II. Stress and self-directed behaviour. Ethology 104, 148-160.

Chamove, A.S., Anderson, J.R., 1989. Examining environmental enrichment. In: Segal, E.F. (Ed.), Housing, Care and Psychological Well-Being of Captive and Laboratory Primates. Noyes Publications, New Jersey, USA, pp. $183-199$.

Davis, H., Levine, S., 1982. Predictability, control and the pituitary-adrenal response in rats. J. Comp. Physiol. Psychol. 96, 393-404.

de Monte, M., Anderson, J.R., Charbonnier, H., 1992. Self-aggression in stumptail macaques: effects of frustration and social partners. Primates 33, 115-120.

de Waal, F.B.M., Hoekstra, J.A., 1980. Contexts and predictability of aggression in chimpanzees. Anim. Behav. 28, 929937.

Diezinger, F., Anderson, J.R., 1986. Starting from scratch: a first look at a "displacement activity" in group-living rhesus monkeys. Am. J. Primatol. 11, 117-124.

Dess, N.K., Linwick, D., Patterson, J., Overmier, J.B., Levine, S., 1983. Immediate and proactive effects of controllability and predictability on plasma cortisol responses to shocks in dogs. Behav. Neurosci. 97, 1005-1016.

Falk, J.L., 1971. The nature and determinants of adjunctive behavior. Physiol. Behav. 6, 577-588.

Falk, J.L., 1977. The origin and functions of adjunctive behaviour. Anim. Learn. Behav. 5, 325-335.

Fraser, D., Matthews, L.R., 1997. Preference and motivation testing. In: Appleby, M.C., Hughes, B.O. (Eds.), Animal Welfare. CAB International, Oxon, UK, pp. 159-173.

Furedy, J.J., Biederman, G.B., 1976. Preference for signaled shock phenomenon: direct and indirect evidence for modifiability factors in the shuttlebox. Anim. Learn. Behav. 4, 1-5.

Garcia, J., Koelling, R.A., 1966. Relation of cue to consequence in avoidance learning. Psychonom. Sci. 4, $123-124$.

Gliner, J.A., 1972. Predictable vs. unpredictable shock: preference behavior and stomach ulceration. Physiol. Behav. 9, 693-698.

Hanson, J.D., Larson, M.E., Snowden, C.T., 1976. The effects of control over high intensity noise on plasma cortisol levels in rhesus monkeys. Behav. Biol. 16, 333-340.

Harris, R.B.S., Mitchell, T.D., Simpson, J., Redmann Jr., S.M., Youngblood, B.D., Ryan, D.H., 2002. Weight loss in rats exposed to repeated acute restraint stress is independent of energy or leptin status. Am. J. Physiol. 282, R77R88.

Hennessy, J.W., King, M.G., McClure, T.A., Levine, S., 1977. Uncertainty, as defined by the contingency between environmental events, and the adrenocortical response of the rat to electric shock. J. Comp. Physiol. Psychol. 91, 1447-1460.

Higa, J.J., Staddon, J.E.R., 1997. Dynamic models of temporal control in animals. In: Bradshaw, C.M., Szabadi, E. (Eds.), Time and Behaviour. Elsevier, Amsterdam, The Netherlands.

Hosey, G.R., 2000. Zoo animals and their human audiences: what is the visitor effect? Anim. Welf. 9, $343-357$.

Hughes, B.O., Duncan, I.J.H., 1988. The notion of ethological 'need', models of motivation and animal welfare. Anim. Behav. 36, 1696-1707.

Hollis, K.L., 1982. Pavlovian conditioning of signal-centered action patterns and autonomic behaviour: a biological analysis of function. Adv. Stud. Behav. 12, 1-64.

Inglis, I.R., 1983. Towards a cognitive theory of exploratory behaviour. In: Archer, J., Birke, L. (Eds.), Exploration in Animals and Humans. Van Nostrand Reinhold, London, UK, pp. 72-116.

Jenny, S., Schmid, H., 2002. Effect of feeding boxes on th behaviour of stereotyping Amur tigers (Panthera tirgis altaica) in the Zurich Zoo, Zurich, Switzerland. Zool. Biol. 21, 573-584.

Johannesson, T., Ladewig, J., 2000. The effect of irregular feeding times on the behaviour and growth of dairy calves. Appl. Anim. Behav. Sci. 69, 103-111.

Jordan, T.C., Coe, C.L., Patterson, J., Levine, S., 1984. Predictability and coping with separation in infant squirrel monkeys. Behav. Neurosci. 98, 556-560.

Kavanau, J.L., 1963. Compulsory regime and control of environment in animal behaviour. I. Wheel -running. Behavior 20, 251-281. 
Krishnamurthy, R., 1994. Vocalizations of captive François' langurs linked to stereotypy and possible stress. Folia Primatol. 63, 91-95.

Lambeth, S., Bloomsmith, M., Baker, K., Perlman, J., Hook, M., Schapiro, S., 2001. Control over videotape enrichment for socially housed chimpanzees: subsequent challenge tests. Am. J. Primatol. 54 (Suppl. 1), $62-63$ (abstract).

Laule, G., 1999. Training laboratory animals. In: Poole, T. (Ed.), The UFAW handbook on the care and management of laboratory animals. Blackwell Science Ltd., Oxon, UK, pp. 21-27.

Laule, G., Desmond, T., 1998. Positive reinforcement training as an enrichment strategy. In: Sheperdson, D.H., Mellen, J.D., Hutchins, M. (Eds.), Second Nature: Environmental Enrichment for Captive Animals. Smithsonian Institute Press, Washington, DC, USA, pp. 302-313.

Levine, S., Goldman, L., Coover, G.D., 1972. Expectancy and the pituitary-reward system. In: Proceedings of the CIAB Foundation Symposia on Physiology, Emotion and Psychosomatic Illness, Elsevier, Amsterdam, The Netherlands, vol. 8, pp. 281-296.

Lindburg, D.G., 1998. Enrichment of captive mammals through provisioning. In: Shepherdson, D.J., Mellen, J.D., Hutchins, M. (Eds.), Second Nature: Environmental Enrichment for Captive Animals. Smithsonian Institute Press, Washington, USA, pp. 262-276.

Line, S.W., Clarke, A.S., Markowitz, H., Ellman, G., 1990. Responses of female rhesus macaques to an environmental enrichment apparatus. Lab. Anim. 24, 213-220.

Line, S.W., Markowitz, H., Morgan, K.N., Strong, S., 1991. Effects of cage size and environmental enrichment on the behavioral and physiological responses of rhesus macaques to the stress of daily events. In: Novak, M.A., Petto, A.J. (Eds.), Through the Looking Glass: Issues of Psychological Well-Being in Captive Nonhuman Primates. American Psychological Association, Washington DC, USA, pp. 160-179.

Lockard, J.S., 1963. Choice of warning signal or no warning signal in an unavoidable shock situation. J. Comp. Physiol. Psychol. 56, 526-530.

Luine, V., Martinez, C., Villegas, M., Magariños, A.M., McEwen, B.S., 1996. Restraint stress reversibly enhances spatial memory performance. Physiol. Behav. 59, 27-32.

Lykken, D.T., Tellegen, A., 1974. On the validity of the preception hypothesis. Psychophysiology 11, $125-132$.

Maier, S.F., 1984. Learned helplessness and animal models of depression. Prog. Neuro-Psychoph. 8, 435-446.

Maestripieri, D., Schino, G., Aureli, F., Troisi, A., 1992. A modest proposal: displacement activities as an indicator of emotions in primates. Anim. Behav. 44, 967-979.

Markowitz, H., 1982. Behavioral Enrichment in the Zoo. Van Nostrand Reinhold, New York, USA.

Mason, G.J., 1993. Forms of stereotypic behaviour. In: Lawrence, A.B., Rushen, J. (Eds.), Stereotypic Animal Behaviour: Fundamentals and Applications to Welfare. CAB International, Oxon, UK, pp. 7-40.

Mason, G.J., Mendl, M., 1993. Why is there no simple way of measuring animal welfare. Anim. Welf. 2, 301-319.

Mason, G.J., Latham, N.R., 2004. Can't stop, won't stop: is stereotypy a reliable animal welfare indicator? Anim. Welf. 13 (Suppl.), S57-S69.

Mason, G., Clubb, R., Latham, N., Vickery, S., 2007. Why and how should we use environmental enrichment to tackle stereotypic behaviour? Appl. Anim. Behav. Sci. 102, 163-188.

Miller, R.R., Greco, C., Vigorito, M., Marlin, N.A., 1983. Signaled tailshock is perceived as similar to a stronger unsignaled tailshock: implications for a functional analysis of classical conditioning. J. Exp. Psychol. Anim. B 9, 105-131.

Miller, S.M., 1979. Controllability and human stress: method, evidence and theory. Behav. Res. Ther. 17, $287-304$.

Mineka, S., Gunnar, M., Champoux, M., 1986. Control and early socioemotional development: infant rhesus monkeys reared in controllable versus uncontrollable environments. Child Dev. 57, 1241-1256.

Mineka, S., Hendersen, R.W., 1985. Controllability and predictability in acquired motivation. Annu. Rev. Psychol. 36, 495-529.

Mineka, S., Kihlstrom, J.F., 1978. Unpredictable and uncontrollable events: a new perspective on experimental neurosis. J. Exp. Psychol. 87, 256-271.

Mistlberger, R.E., 1994. Circadian food-anticipatory activity: formal models and physiological mechanisms. Neurosci. Biobehav. R. 18, 171-195.

Mistlberger, R.E., Rusak, B., 1987. Palatable daily meals entrain anticipatory activity rhythms in free-feeding rats: dependence on meal size and nutrient content. Physiol. Behav. 41, 219-226.

Mowrer, O.H., 1960. Learning Theory and Symbolic Processes. Wiley, New York, USA.

Nickels, J.B., Cramer, K.M., Gural, D.M., 1992. Toward unconfounding prediction and control: predictionless control made possible. Can. J. Behav. Sci. 24, 156-170.

Novak, M.A., Drewson, K.H., 1989. Enriching the lives of captive primates: issues and problems. In: Segal, E.F. (Ed.), Housing, Care and Psychological Well-Being of Captive and Laboratory Primates. Noyes Publications, New Jersey, USA, pp. 161-182. 
Overmier, J.B., Patterson, J., Wielkiewicz, R.M., 1980. Environmental contingencies as sources of stress in animals. In: Levine, S., Ursin, H. (Eds.), Coping and Health. Plenum Press, New York, USA, pp. 1-38.

Pavlov, I.P., 1927. Conditioned Reflexes: An Investigation of the Physiological Activity of the Cerebral Cortex. Oxford University Press, London, UK.

Perkins Jr., C.C., 1955. The stimulus conditions which follow learned responses. Psychol. Rev. 62, 341-348.

Perkins Jr., C.C., 1968. An analysis of the concept of reinforcement. Psychol. Rev. 75, 155-172.

Phillippi-Falkenstein, K., Clarke, M.R., 1992. Procedure for training corral-living rhesus-monkeys for fecal and bloodsample collection. Lab. Anim. Sci. 42, 83-85.

Pitman, D.L., Natelson, B.H., Ottenweller, J.E., McCarty, R., Pritzel, T., Tapp, W.N., 1995. Effects of exposure to stressors of varying predictability on adrenal function in rats. Behav. Neurosci. 109, 767-776.

Prescott, M.J., Buchanan-Smith, H.M. (Eds.), 2003. Training nonhuman primates using positive reinforcement techniques. J. Appl. Anim. Welf. Sci., 6.

Prokasky, W.F., 1956. The acquisition of observing responses in the absence of differential external reinforcement. J. Comp. Physiol. Psychol. 49, 131-134.

Reinhardt, V., 2003. Working with rather than against macaques during blood collection. J. Appl. Anim. Welf. Sci. 6, 189198.

Reneerkens, J., Piersma, T., Ramenofsky, M., 2002. An experimental test of the relationship between temporal variability of feeding opportunities and baseline levels of corticosterone in a shorebird. J. Exp. Zool. 293, 81-88.

Reynolds, V., Luscombe, G.P., 1969. Social Behavior of Chimpanzees in an Open Environment. 657 1st Aeromedical Research Laboratory, Aerospace Medical Division, United States Air Force.

Richelle, M., Lejeune, H., 1980. Time in Animal Behaviour. Pergamon Press, Oxford, UK.

Roberts, W.A., 1998. Principles of Animal Cognition. McGraw-Hill, USA.

Saldanha, C.J., Schlinger, B.A., Clayton, N.S., 2000. Rapid effects of corticosterone on cache recovery in mountain chickadees (Parus gambelii). Horm. Behav. 37, 109-115.

Salzen, E.A., 1962. Imprinting and fear. Symp. Zool. Soc. Lon. 8, 199-218.

Sambrook, T.D., Buchanan-Smith, H.M., 1997. Control and complexity in novel object enrichment. Anim. Welf. 6, 207216.

Sandi, C., Venero, C., Gauza, C., 1996. Novelty-related rapid locomotor effects of corticosterone in rats. Eur. J. Neurosci. 84, 794-800.

Schulz, R., 1976. Effects of control and predictability on the physical and psychological well-being of the institutionalized aged. J. Pers. Soc. Psychol. 33, 563-573.

Scott, L., 1990. Training non-human primates: meeting their behavioural needs. In: UFAW (Eds.), Animal Training: a Review and Commentary. Universities Federation for Animal Welfare, Herts, UK, pp. 129-133.

Seligman, M.E.P., 1968. Chronic fear produced by unpredictable electric shock. J. Comp. Physiol. Psychol. 66, $402-411$.

Seligman, M.E.P., 1975. Helplessness: on depression. In: Development and Death, W H Freeman, San Francisco, USA.

Seligman, M.E.P., Meyer, P., 1970. Chronic fear and ulcers in rats as a function of the unpredictability of safety. J. Comp. Physiol. Psychol. 73, 202-207.

Shepherdson, D., 1989. Environmental enrichment. Ratel 16, 4-9.

Shepherdson, D.J., Carlstead, K., Mellen, J.D., Seidensticker, J., 1993. Influence of food presentation on the behaviour of small cats in confined environments. Zool. Biol. 12, 203-216.

Tarou, L.R., Bashaw, M.J., 2007. Maximising the effectiveness of environmental enrichment. Appl. Anim. Behav. Sci. 102, 189-204.

Taylor, P.E., Haskell, M., Appleby, M.C., Waran, N.K., 2002. Perception of time duration by domestic hens. Appl. Anim. Behav. Sci. 76, 41-51.

Tsuda, A., Tanaka, M., Hirai, H., Paré, W.P., 1983. Effects of coping behavior on gastic lesions in rats as a function of predictability of shock. Jpn. Psychol. Res. 25, 9-15.

Tsuda, A., Tanaka, M., Nishikawa, T., Hirai, H., Paré, W.P., 1984. Effects of unpredictability versus loss of predictability of shock on gastric lesions in rats. Physiol. Psychol. 11, 287-290.

van Rooijen, J., 1991. Predictability and boredom. Appl. Anim. Behav. Sci. 31, 283-287.

Wagner, A.R., 1959. The role of reinforcement and nonreinforcement in an 'apparent frustration effect'. J. Exp. Psychol. 57, 130-136.

Waitt, C., Buchanan-Smith, H.M., 2001. What time is feeding? How delays and anticipation of feeding schedules affect stump-tailed macaque behaviour. Appl. Anim. Behav. Sci. 75, 75-85.

Waitt, C., Buchanan-Smith, H.M., Morris, K., 2001. The impact of human activity on laboratory primates. In: Hare, V.J., Worley, K.E., Myers, K. (Eds.), Proceedings of the 4th International Conference on Environmental Enrichment, The Shape of Enrichment, Inc., San Diego, USA, pp. 121-132. 
Wasserman, F.E., Cruikshank, W.W., 1983. The relationship between time of feeding and aggression in a group of captive hamadryas baboons. Primates 24, 432-435.

Weinberg, J., Levine, S., 1980. Psychobiology of coping in animals: the effects of predictability. In: Levine, S., Ursin, H. (Eds.), Coping and Health. Plenum Press, New York, USA, pp. 39-59.

Weiss, J.M., 1968. Effects of coping responses on stress. J. Comp. Physiol. Psychol. 65, 251-260.

Weiss, J.M., 1971a. Effects of coping behavior in different warning signal conditions on stress pathology in rats. J. Comp. Physiol. Psychol. 77, 1-13.

Weiss, J.M., 1971b. Effects of punishing the coping response (conflict) on stress pathology in rats. J. Comp. Physiol. Psychol. 77, 14-21.

Weiss, J.M., 1972. Psychological factors in stress and disease. Sci. Am. 226, 104-113.

Wiepkema, P.R., Koolhaas, J.M., 1993. Stress and animal welfare. Anim. Welf. 2, 195-218.

Wilson, W.L., Wilson, C.C., 1968. Aggressive Interactions of Captive Chimpanzees Living in a Semi Freeranging Environment. Technical Report No. ARL-TR-68-9. Aeromedical Research Laboratory, Mew Mexico.

Wingfield, J.C., Ramenofsky, M., 1999. Hormones and the behavioral ecology of stress. In: Balm, P.H.M. (Ed.), Stress Physiology in Animals. Sheffield Academic Press, Sheffield, UK, pp. 1-51.

Wykoff, L.B., 1952. The role of observing responses in discrimination learning; part 1. Psychol. Rev. 59, 431-442.

Young, R.J., 2003. Environmental Enrichment for Captive Animals. Blackwell Science, Oxford. 